\title{
Trojan Horse Method experiments with radioactive ion beams
}

\author{
Marisa Gulino ${ }^{1,2, \star}$, Silvio Cherubini ${ }^{1,3, \star \star}$, Giuseppe Gabriele Rapisarda ${ }^{1}$, Shigeru Kubono ${ }^{4}$ \\ Livio Lamia ${ }^{1,3}$, Marco La Cognata ${ }^{1}$, Rosario Gianluca Pizzone ${ }^{1}$, Hidetoshi Yamaguchi ${ }^{5}$, Seya \\ Hayakawa $^{5}$, Yasuo Wakabayashi ${ }^{5}$, Naohito Iwasa $^{6}$, Seigo Kato ${ }^{7}$, Tetsuro Komatsubara ${ }^{8}$, Takashi \\ Teranishi $^{9}$, Alain Coc $^{10}$, Nicolas de Séréville ${ }^{11}$, Fairouz Hammache ${ }^{11}$, Gabor Kiss ${ }^{12}$, Shawn \\ Bishop $^{13}$, Dam Nguyen Binh ${ }^{5,14}$, Brian Roeder ${ }^{15}$, Livius Trache ${ }^{16}$, Robert Tribble ${ }^{15,17}$, Roberta \\ Spartà ${ }^{1}$, Iolanda Indelicato ${ }^{1}$, and Claudio Spitaleri ${ }^{1,3}$ \\ ${ }^{1}$ INFN-LNS, Catania, Italy \\ ${ }^{2}$ Università di Enna Kore, Enna, Italy \\ ${ }^{3}$ Dipartimento di Fisica e Astronomia, Università di Catania, Catania, Italy \\ ${ }^{4}$ RIKEN Nishina Center, 2-1 Hirosawa, Wako, Saitama 351-0198, Japan \\ ${ }^{5}$ CNS, University of Tokyo, Wako Branch, 2-1 Hirosawa, Wako, Saitama 351-0198, Japan \\ ${ }^{6}$ Department of Physics, Tohoku University, 6-6 Aoba, Sendai, Miyagi 980-8578, Japan \\ ${ }^{7}$ Department of Physics, Yamagata University, Yamagata 990-8560, Japan \\ ${ }^{8}$ Rare Isotope Science Project, IBS, Yuseong-daero, Yuseong-gu, Daejeon 305-811, Korea \\ ${ }^{9}$ Department of Physics, Kyushu University, Fukuoka 812-8581, Japan \\ ${ }^{10}$ Centre de Spectrométrie Nucléaire et de Spectrométrie de Masse, IN2P3, F-91405 Orsay, France \\ ${ }^{11}$ Institut de Physique Nucléaire, IN2P3, F-91405 Orsay, France \\ ${ }^{12}$ Institute for Nuclear Research (MTA-ATOMKI), Debrecen, Hungary \\ ${ }^{13}$ TUM, Garching, Germany \\ ${ }^{14} 30 \mathrm{MeV}$ Cyclotron Center, Tran Hung Dao Hospital, Hoan Kiem District, Hanoi, Vietnam \\ ${ }^{15}$ Texas A\& M Univ, Inst Cyclotron, College Stn, TX 77843 USA \\ ${ }^{16}$ IFIN HH, Bucharest, Romania \\ ${ }^{17}$ Brookhaven Natl Lab, Upton, NY 11973 USA
}

\begin{abstract}
The Trojan Horse Method (THM) is an indirect method that allows to get information about a two body reaction cross-section even at very low energy, avoiding the suppression effects due to the presence of the Coulomb barrier. The method requires a very accurate measurement of a three body reaction in order to reconstruct the whole kinematics and discriminate among different reaction mechanisms that can populate the same final state. These requirements hardly match with the typical low intensity and large divergence of radioactive ion beams (RIBs), and experimental improvements are mandatory for the applicability of the method. The first reaction induced by a radioactive ion beam studied by applying the THM was the ${ }^{18} \mathrm{~F}(p, \alpha){ }^{15} \mathrm{O}$. Two experiments were performed in two different laboratories and using different experimental set-ups. The two experiments will be discussed and some results will be presented.
\end{abstract}

\footnotetext{
$\star$ e-mail: gulino@1ns.infn.it

$\star \star$ e-mail: cherubini@lns.infn.it
} 


\section{Introduction}

The Trojan Horse Method (THM) has been recently extended to reactions induced by radioactive ion beams (RIB).

The method allows to get information about a nuclear reaction cross-section having two body in the final state, $A+x \rightarrow b+c$, by studying an appropriate reaction with three body in the final state, $A+a \rightarrow b+c+s$. The nucleus $a$, called Trojan Horse nucleus, must have a strong cluster structure $x$-s. So, it is possible to have events in which the nucleus $a$ breaks-up inside the nuclear field of the nucleus $A$, the cluster $x$ interacts with the nucleus $A$ through the $A+x \rightarrow b+c$ reaction, while the cluster $s$ acts as a spectator. If this is the case, the cross section of the $A+a \rightarrow b+c+s$ reaction can be easily factorized and used to deduce that one of the $A+x \rightarrow b+c$ reaction $[1,2]$.

In a THM experiment, once the Trojan Horse nucleus $a$ has been chosen, the energy and the angular position of at least two out of the three ejected particles in the $A+a \rightarrow b+c+s$ reaction must be detected. So, making an hypothesis about the nature of the undetected nucleus, it is possible to reconstruct the whole kinematics [5] and to select the reaction channel of interest. Moreover, to apply the factorization of the cross section of the three body reaction so inferring information about the reaction of interest, only the events proceeding through a quasi free reaction mechanism must be considered.

In order to proceed through these data analysis steps, some features should be taken into account. First of all, the kinematic is very sensitive to the angles of the tracks of the outgoing products. For this reason, in the usual THM experiments, the beam spot diameter is reduced to 1 or $2 \mathrm{~mm}$, the thickness of the target is kept as thin as possible, and position sensitive detectors having a resolution of the order of millimetre are used in the experimental set-up. Moreover, as the quasi-free events are a small fraction of the whole statistics (generally of the order of $10 \%$, depending strongly on the reaction), it is mandatory to collect enough events to obtain reasonable statistical errors.

All these requirements hardly match with the typical low intensity, presence of contaminants and large divergence of RIBs. To overcome these difficulties, more efficient experimental set-up must be used when RIBs are involved in THM experiments.

\section{The ${ }^{18} \mathbf{F}(p, \alpha){ }^{15} \mathbf{O}$ reaction}

The first reaction induced by a RIB that has been studied by applying the THM is the ${ }^{18} \mathrm{~F}(p, \alpha){ }^{15} \mathrm{O}$. This reaction is important to understand the novae explosion phenomena. The $\gamma$-ray emission following the novae explosion is dominated by the $511 \mathrm{keV}$ energy line, coming from the annihilation of positrons produced by the decay of radioactive nuclei. Among them, the ${ }^{18} \mathrm{~F}$ is expected to be abundant in the novae environment, and its lifetime well matchs the timescale for novae ejects to become transparent to $\gamma$-ray emission. It is then important to know the rate of the nuclear reactions producing and destroying ${ }^{18} \mathrm{~F}$.

At the relevant temperatures, the cross section of the ${ }^{18} \mathrm{~F}(p, \alpha){ }^{15} \mathrm{O}$ reaction that destroys ${ }^{18} \mathrm{~F}$ is dominated by the presence of several levels in the ${ }^{19} \mathrm{Ne}$ compound nucleus around the ${ }^{18} \mathrm{~F}$-p threshold. For this reason, it is especially important to have a good reconstruction of the ${ }^{18} \mathrm{~F}$-p relative energy. Moreover, the tails of some subthreshold levels are expected to contribute to the resonance cross section, and interference effects between excited states having the same spin can also occur.

To infer information on the ${ }^{18} \mathrm{~F}(p, \alpha){ }^{15} \mathrm{O}$ reaction in the low interesting energy region, the three body $d\left({ }^{18} \mathrm{~F}, \alpha^{15} \mathrm{O}\right) n$ reaction was studied by using a ${ }^{18} \mathrm{~F}$ beam impinging on a $\mathrm{CD}_{2}$ solid target. Two different experiments were performed in two different laboratories and using different experimental set-up.

The first experiment was performed at the CRIB facility in RIKEN, Japan [3]. The second one was performed using the MARS spectrometer installed at TAMU, USA [4]. 

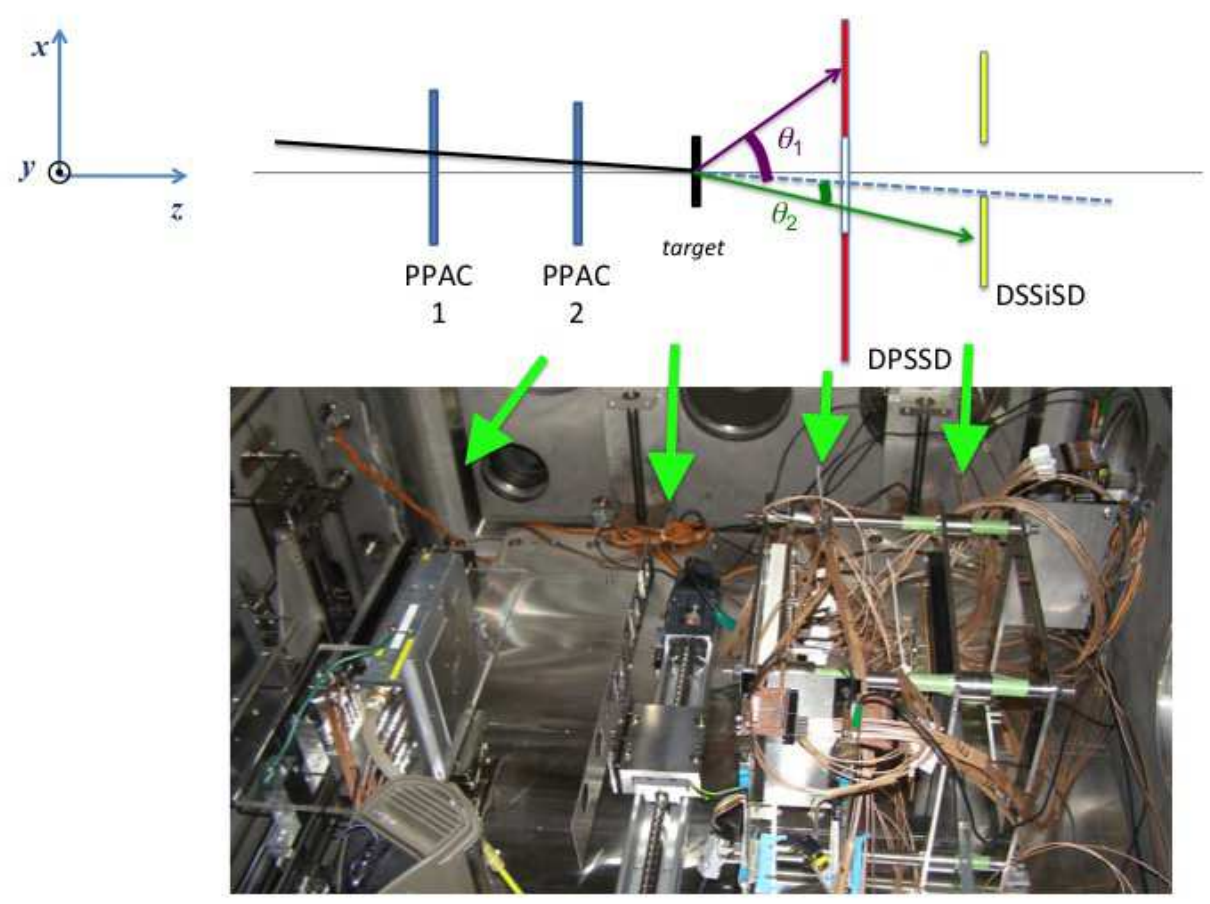

Figure 1. Scheme and photo of the experimental set-up used for the ${ }^{18} \mathrm{~F}+d$ experiment performed at CRIB, RIKEN, Japan (upper view). The beam line is in the $\mathrm{z}$ axis; PPAC 1 and PPAC 2 track the effective beam trajector event by event; DPSSD is the plane containing 8 double position sensitive Si detectors by Hamamatsu; DSSiSD is a couple of double sided Si strip detectors. Note that the angles of the ejected particles are reconstructed with respect to the measured beam track event by event.

In order to partially overcome the problems arising from the typical low intensity, presence of contaminants and large divergence of RIBs, the experimental set-up used in both the experiments are very different from those used in standard THM experiments. Indeed, detectors covering a large solid angle were used to measure the energy and the angle of the ejected particles. In particular, in the CRIB experiment a pair of a double sided multistrip detectors (DSSiSD), manufactured by Micron Semiconductor (16 strips per side, width $3 \mathrm{~mm}$ ) and placed at $287 \mathrm{~mm}$ from the target, were used to detect the heaviest ejectile. The other ejectile was detected by using the ASTRHO (A Silicon Array for TRojan HOrse) modular system of INFN Laboratori Nazionali del Sud. In the configuration used for this experiment, ASTRHO was equipped with 8 bidimensional position sensitive detectors $(45 \times 45$ $\mathrm{mm}^{2}$, spatial resolution $1 \mathrm{~mm}$ ), manufactured by Hamamatsu Photonics and placed at $150 \mathrm{~mm}$ from the target. For each detector the $x$ and $y$ position and the energy of the impinging particles were recorded together with the time of flight. A sketch of the experimental set-up is shown in fig. 1.

The set-up used in the experiment performed at TAMU consists of two position sensitive detectors (PSDs, type X1,16 strips each), placed symmetrically at $340 \mathrm{~mm}$ from target and used to detect the heaviest ejectile, and the TECSA array[6], made up of 8 YY1-300 Micron detectors (each one with 16 arch strips), placed at $190 \mathrm{~mm}$ from target used. In fig. 2 a schematic view of the experimental 

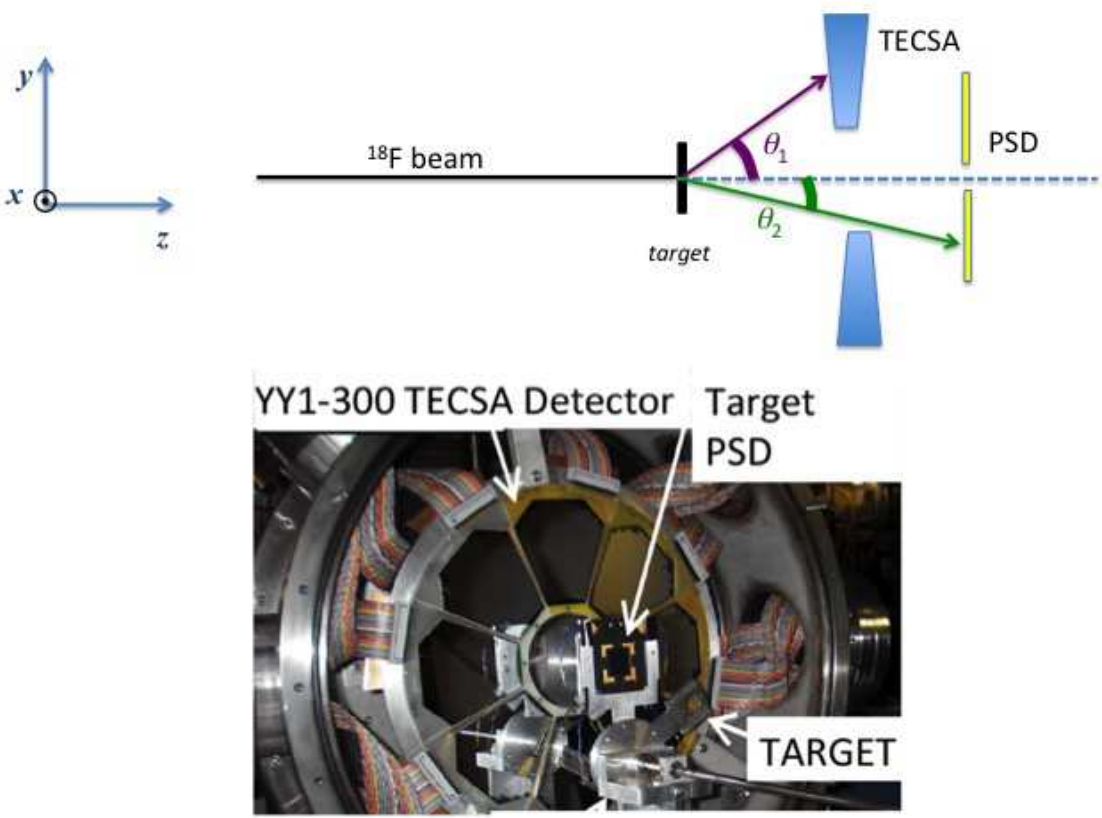

Figure 2. Scheme and photo of the experimental set-up used for the ${ }^{18} \mathrm{~F}+d$ experiment performed at Mars, TAMU, USA. The beam line is the $\mathrm{z}$ axis; TECSA is the Si strip detector plane containing 8 annular sectors; PSD is a couple of position sensitive $\mathrm{Si}$ detectors.

set-up is shown.

The main difference between the two experimental set-ups comes from the use of a beam tracker in the CRIB experiment consisting of two PPAC detectors placed at $510 \mathrm{~mm}$ and $107 \mathrm{~mm}$ before the target position and having a spatial resolution of $1 \mathrm{~mm}$ (see fig.1). This assures the reconstruction of the beam track with a geometrical angular resolution of $0.14^{\circ}$. The emission angles of the tracks of the ejectiles were then reconstructed with respect to the measured beam track to improve the angular resolution (see fig. 1).

Some details of the two experiments can be found in the table 1.

The different energy resolution achieved in the two experiments can be observed in fig. 3 where the bare nuclear cross section $\left(\sigma^{N}\right)$, free of Coulomb suppression effects, of the ${ }^{18} \mathrm{~F}(\mathrm{p}, \alpha){ }^{15} \mathrm{O}$ reaction is reported. The black dots report the data measured at CRIB, while the blue open squares are from the TAMU experiment. The full line is a multigaussian fit performed using data from the RIKEN experiment. The energies of the populated states of the ${ }^{19} \mathrm{Ne}$ are reported in the figure. The full line is a multigaussian fit performed on data from the RIKEN experiment. The energies in $\mathrm{keV}$ of the populated states of the ${ }^{19} \mathrm{Ne}$ are reported in the figure. The width of each gaussian was fixed by using the experimental resolution calculated by using a Monte Carlo simulation. A very interesting application of the THM to RIBs induced reactions is the study of neutron induced reactions on radioactive isotopes, even if they have a short lifetime [7-9]. Preliminary analysis of the ${ }^{18} \mathrm{~F}(\mathrm{n}, \alpha){ }^{15} \mathrm{~N}$ has been 
Table 1. Experimental details of the experiments performed at RIKEN- Japan and TAMU - USA for the measurement of the ${ }^{18} \mathrm{~F}(\mathrm{p}, \alpha)$ reaction

\begin{tabular}{l|lc}
\hline & CRIB - RIKEN - Japan & \multicolumn{2}{c}{ Mars - TAMU - USA } \\
\hline \multirow{3}{*}{ Beam energy } & \multicolumn{2}{c}{${ }^{18} \mathrm{~F}$ beam } \\
\cline { 2 - 3 } Beam spot & $47.9 \mathrm{MeV}(\mathrm{FWHM} 1.9 \mathrm{MeV})$ & $52 \mathrm{MeV}(\mathrm{FWHM} 2.6 \mathrm{MeV})$ \\
Beam purity & tracked & $3 \mathrm{~mm} \times 5 \mathrm{~mm}$ \\
Beam intensity & $98 \%$ & $94 \%$ \\
& $5 \times 10^{5} \mathrm{pps}$ & $3-4 \times 10^{5} \mathrm{pps}$ \\
\cline { 2 - 3 } target thickness & $150-200 \mu \mathrm{g} / \mathrm{cm}^{2}$ & $\mathrm{CD}_{2}$ target \\
\hline \multirow{4}{*}{ angular range } & \multicolumn{2}{c}{$400-800 \mu \mathrm{g} / \mathrm{cm}^{2}$} \\
\cline { 2 - 3 } angular resolution & $2^{\circ}-11^{\circ}$ & Detectors \\
(geometrical) & $11^{\circ}-31^{\circ}$ & $3^{\circ}-12^{\circ}$ \\
energy resolution & $0.2^{\circ}$ & $15^{\circ}-40^{\circ}$ \\
\hline
\end{tabular}

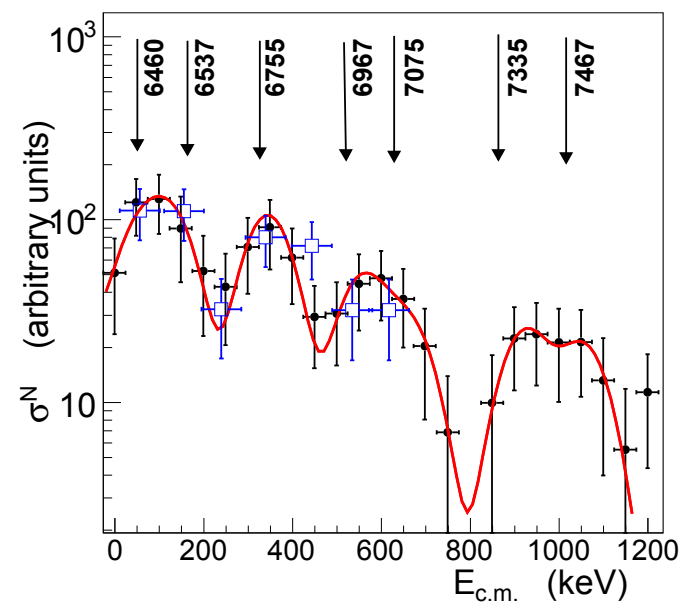

Figure 3. Bare nuclear cross section for the ${ }^{18} \mathrm{~F}(\mathrm{p}, \alpha){ }^{15} \mathrm{O}$ reaction measured by using the THM. The full dots refer to the RIKEN experiment while the blue open square are from the TAMU experiment. The full line is a multigaussian fit performed on data from the RIKEN experiment. The energies in $\mathrm{keV}$ of the populated states of the ${ }^{19} \mathrm{Ne}$ are reported in the figure.

performed using the experimental data from the CRIB experiment. The extracted differential bare nuclear cross-section is shown in fig. 4.

\section{Conclusions}

The THM was extended to study reactions induced by RIBs. Even with the use of indirect methods like THM, the measurement of cross sections of interest for nuclear astrophysics remains one of the most difficult tasks in nuclear physics, especially if one has to use RIBs. Indeed, low beam intensity, 


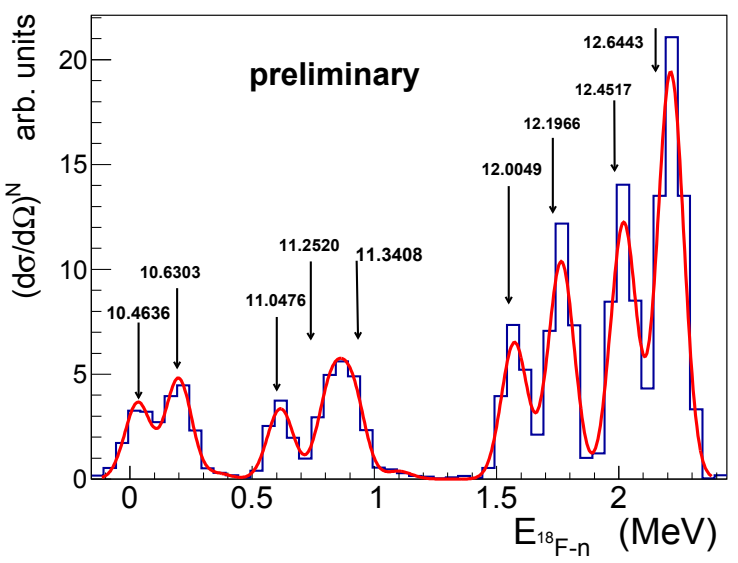

Figure 4. Bare nuclear cross section for the ${ }^{18} \mathrm{~F}(\mathrm{n}, \alpha){ }^{15} \mathrm{~N}$ reaction measured with the THM at the CRIB facility, RIKEN, Japan.

large divergence and presence of contaminants are added on the difficulties of measuring the low cross sections, which are typical of astrophysical nuclear processes.

The THM has been successfully used to infer pieces of information about the ${ }^{18} \mathrm{~F}(\mathrm{p}, \alpha){ }^{15} \mathrm{O}$ by studying the three body reaction ${ }^{18} \mathrm{~F}\left(\mathrm{~d}, \alpha{ }^{15} \mathrm{O}\right)$ n. Two experiments were performed in two different laboratories and using different experimental set-up, and similar results have been obtained. The comparison has stressed the fundamental role played by the beam tracking system to improve the energy resolution of the obtained cross section.

Calculations on the rate of the ${ }^{18} \mathrm{~F}(\mathrm{p}, \alpha){ }^{15} \mathrm{O}$ reaction performed by using a modified R-matrix code have shown an higher destruction rate in the temperature range of interest for the novae. Consequently, the ${ }^{18} \mathrm{~F}$ content in novae is decreased by a factor $2[10]$.

\section{References}

[1] C. Spitaleri. in Proceedings of the Fifth Hadronic Physics Winter Seminar, Folgaria - TN, Italy, Ed. World Scientific, Singapore, 1990.

[2] S. Cherubini et al., ApJ 457, 855, (1996)

[3] S. Cherubini et al., Phys. Rev. C 92, 015805 (2015)

[4] R.G. Pizzone et al., EPJA, 5224 (2016)

[5] G.G. Ohlsen NIM 37, 240, (1965)

[6] B.T. Roeder et al., Nucl. Instrum. Methods A 634, 71 (2011)

[7] M. Gulino et al., J. Phys. G: Nucl. Part. Phys. 37, 125105 (2010)

[8] M. Gulino et al., Phys. Rev. C 87, 012801 (2013)

[9] M. Gulino et al., Nuovo Cimento C - Coll. and Comm. in Phys. 39(5), 369 (2016)

[10] M. La Cognata at al., ApJ 846, 65, (2017) 